\title{
Aplicación de $\mathrm{MgSO}_{4} \cdot 7 \mathrm{H}_{2} \mathrm{O}$ y cenizas volantes como refuerzo en la matriz de unidades cerámicas macizas*
}

\author{
Karol Lizeth Roa Bohórquez ${ }^{* *}$ \\ Ricardo Alfonso Paredes Roa ${ }^{* * *}$ \\ Luis Ángel Lara González $z^{* * *}$
}

Recibido: 09/07/2016 • Aceptado: 23/10/2017

DOI: 10.22395/rium.v17n32a2

\begin{abstract}
Resumen
Sereportan las composiciones químicas obtenidas por FRX dearcillay cenizas volantes producto de la combustión de carbón en un horno tipo colmena. Se seleccionaron las cantidades de arcilla, ceniza volante y sulfato de magnesio $\left(\mathrm{MgSO}_{4} \cdot 7 \mathrm{H}_{2} \mathrm{O}\right)$ en función de su composición química para lograr una eflorescencia total en la superficie del ladrillo (unidad cerámica maciza). Una vez se conformaron por prensado las unidades de mampostería, se sometieron a tratamiento a $400{ }^{\circ} \mathrm{C}$ durante dos horas con el fin de eliminar la humedad, una vez alcanzaron $950{ }^{\circ} \mathrm{C}$ se cocinan en esta temperatura durante tres horas. Se estableció la composición química de las eflorescencias en la superficie de las probetas por medio de FRX, se analizó morfológicamente el corte transversal de un ladrillo, y por último, las probetas se sometieron a ensayos de compresión para determinar su resistencia y de absorción máxima de agua, teniendo en cuenta la norma técnica colombiana NTC 4205.
\end{abstract}

Palabras clave: cerámica; arcillas; ladrillos; cenizas volantes.

Artículo de revisión. Proyecto de investigación de capital semilla SGI 1997 financiado por Dirección de Investigación de la Universidad Pedagógica y Tecnológica de Colombia (UPTC).

** Estudiante de Ingeniería Industrial, integrante del Grupo de Investigación Geoquímica Ambiental (GEAM), Universidad Pedagógica y Tecnológica de Colombia (UPTC). karol.roa@uptc.edu.co

*** Estudiante de Ingeniería Industrial, integrante del Grupo de Investigación Geoquímica Ambiental (GEAM), Universidad Pedagógica y Tecnológica de Colombia (UPTC).ricardo.paredes@uptc.edu.co

**** Integrante del Grupo de Investigación Geoquímica Ambiental (GEAM), Universidad Pedagógica y Tecnológica de Colombia (UPTC). luisangel.lara@uptc.edu.co| 


\title{
Application of $\mathrm{MgSO}_{4} \cdot 7 \mathrm{H}_{2} \mathrm{O}$ and Fly Ash as a Reinforcement in the Matrix of Solid Ceramic Units
}

\begin{abstract}
Chemical compositions obtained by XRF from clay and fly ash from coal combustion in a hive furnace are reported. The quantities of clay, fly ash and magnesium sulphate $\left(\mathrm{MgSO}_{4} \cdot 7 \mathrm{H}_{2} \mathrm{O}\right)$ were selected according to their chemical composition to achieve total efflorescence on the surface of the brick (solid ceramic unit). Once the masonry units were formed by pressing, they were treated at $400{ }^{\circ} \mathrm{C}$ for two hours to remove moisture, and once they reached $950^{\circ} \mathrm{C}$, they were cooked at this temperature for three hours. The chemical composition of the efflorescence on the surface of the specimens was established by XRF, the cross section of a brick was morphologically analyzed and, finally, the specimens were subjected to compression tests to determine their resistance and maximum water absorption, taking into account Colombian technical standard NTC 4205.
\end{abstract}

Keywords: ceramics; clays; bricks; fly ashes.

\section{Aplicação de $\mathrm{MgSO}_{4} \cdot 7 \mathrm{H}_{2} \mathrm{O}$ e cinzas voadoras como reforço na matriz de unidades cerâmicas maciças}

\begin{abstract}
Resumo
Relatam-se as composições químicas obtidas por FRX de argila e cinzas voadoras produto da combustão de carvão num forno tipo colmeia. Foram selecionadas as quantidades de argila, cinza voadora e sulfato de magnésio $\left(\mathrm{MgSO}_{4} \cdot 7 \mathrm{H}_{2} \mathrm{O}\right)$ em função de sua composição química para atingir uma eflorescência total na superfície do tijolo (unidade cerâmica maciça). Assim que as unidades de alvenaria foram prensadas, foram submetidas a tratamento a $400{ }^{\circ} \mathrm{C}$ durante duas horas, a fim de eliminar a umidade; uma vez tenham atingido $950{ }^{\circ} \mathrm{C}$, foram cozidas nessa temperatura durante três horas. Foi estabelecida a composição química das eflorescências na superfície das provetas por meio de FRX; logo, foi analisado morfologicamente o corte transversal de um tijolo e, por último, as provetas foram submetidas a ensaios de compressão para determinar sua resistência e absorção máxima de água, considerando a norma técnica colombiana NTC 4205.
\end{abstract}

Palavras-chave: cerâmica; argila; tijolo; cinza voadora. 


\section{INTRODUCCIÓN}

Las unidades cerámicas macizas comúnmente conocidas como ladrillos son uno de los productos cerámicos más comunes en la industria alfarera; su materia prima en mayor composición es la arcilla (elemento matriz) la cual presenta múltiples aplicaciones especializadas, no solo es utilizada en la fabricación de cerámica tradicional, sino que también se usa para la elaboración de nuevos materiales útiles hoy en día [1-3].

La arcilla es un mineral complejo que se encuentra compuesto por aluminosilicatos hidratados, generalmente en presencia de otros minerales como magnesio, hierro, titanio y calcio [4]. De acuerdo con el tipo de ladrillo que se desea, ya sea perforado horizontal, vertical o macizo, su elaboración sigue un proceso básico: trituración de arcillas, mezcla e hidratación, conformado (presión o extrusión), secado al aire libre y cocción de las piezas [5].

Uno de los procesos más importantes es el secado; como las piezas están perdiendo peso debido a la difusión de moléculas de agua hasta la superficie, las partículas de arcilla disminuyen su distancia entre sí generando contracción del material [6-7] por la presencia de sales en el componente hidratante y en el tipo de arcilla utilizada; durante el proceso de evaporación estos minerales se depositan en la superficie y generan manchas blancas [8]. Las eflorescencias constituyen un grave problema para la industria alfarera por su aspecto visual [9]; estas manchas superficiales generalmente de color claro o blanco son generadas principalmente por sales solubles o insolubles [10-12].

Una de las sales que genera eflorescencias es el sulfato de magnesio [13]; esta sal se genera principalmente en los medios arcillosos debido a su composición; sin embargo, también puede formarse por interacción con los gases del horno [14-15]. En el presente trabajo se busca determinar la influencia que genera una eflorescencia total en la superficie de las unidades cerámicas, con la adición de 5\% de sulfato de magnesio sintético en la mezcla [16] comparándolo con el comportamiento de las unidades sin la adición del sulfato de magnesio.

Por otra parte, en la elaboración de las piezas cerámicas se agregan cenizas volantes, desecho proveniente del proceso de combustión del carbón para la cocción de ladrillos. Debido a la composición química de los polvos de cenizas, el desecho actúa como desgrasante (refuerzo) y aliviana las unidades cerámicas gracias a su baja densidad [17-18].

\section{MATERIALES Y MÉTODOS}

La muestra de arcilla y los polvos de cenizas volantes los cuales son producto de la combustión del carbón en un horno tipo colmena fueron proporcionados por una fábrica ladrillera, ubicada en Sogamoso-Colombia. 
Para determinar la composición química de las materias primas se realizaron fluorescencias de rayos X (FRX) de la muestra de arcilla y los polvos de ceniza, los cuales se llevaron a una granulometría malla 200 para su análisis en el equipo PANalytical MiniPal 2.

Para determinar el límite de plasticidad, el índice plástico y el límite líquido de los polvos de arcilla se siguieron los procedimientos especificados según norma [19 - 20].

El cálculo del límite líquido se realizó con la preparación de tres muestras de 12,8 g, 8,6 g y 9,5 g. A cada muestra se le agregó agua destilada hasta formar una mezcla a punto de moldeo, se depositaron en Cuchara de Casagrande o Cazuela de Casagrande y se golpearon consecutivamente contra la base de la máquina, haciendo girar la manivela, hasta que el surco que previamente se había recortado se cerrara a los 13, 23 y 40 golpes, respectivamente.

Para el límite plástico se preparó una mezcla de agua destilada y suelo arcilloso; mediante moldeo manual se formaron cilindros de $3 \mathrm{~mm}$ de diámetro los cuales se depositaron en un recipiente metálico en reposo durante 24 horas a $80^{\circ} \mathrm{C}$. Según la carta de plasticidad introducida por el Sistema Unificado de Clasificación de Suelos (SUCS), se utilizó el modelo (1) para obtener el índice de plasticidad, reportado en [1]:

$$
I P=0,73(L L-20)
$$

Donde IP es el índice de plasticidad (\%); y $L L$ es el límite líquido (\%) hallado en el ensayo.

Las unidades de mampostería que actuaron de referencia para determinar las influencias de la adición del $\mathrm{MgSO}_{4} \cdot 7 \mathrm{H}_{2} \mathrm{O}$ se elaboraron con una mezcla de $80 \%$ arcilla y $20 \%$ de cenizas volantes. Por otro lado, se elaboraron unidades con una mezcla de $80 \%$ arcilla, $15 \%$ de cenizas volantes y $5 \%$ de sulfato de magnesio $\left(\mathrm{MgSO}_{4} \cdot 7 \mathrm{H}_{2} \mathrm{O}\right)$ para generar las eflorescencias; el proceso de conformado seleccionado para todas las muestras fue prensado. Posteriormente, se pesaron y dejaron secar durante 24 horas a $90{ }^{\circ} \mathrm{C}$. Una vez transcurrido dicho tiempo se pesaron de nuevo y dejaron por 36 horas a la misma temperatura hasta alcanzar una masa constante.

La sinterización de las piezas se llevó a cabo en una mufla eléctrica Thermolyne ref. F6018 marca Thermo Scientific, la cual se programó con una curva de cocción a una rapidez de $5{ }^{\circ} \mathrm{C} / \mathrm{min}$ hasta alcanzar $400{ }^{\circ} \mathrm{C}$, temperatura en la cual se mantuvo durante 2 horas; una vez transcurrido el tiempo se elevó a $950^{\circ} \mathrm{C}$ y se mantuvo en esa temperatura por tres horas más. 
Por medio de fotografía de un corte transversal se realizó el análisis morfológico de las probetas con adición de sulfato de magnesio; la capa blanca de eflorescencias presentada en la superficie se analizó por fluorescencia de rayos X.

Las propiedades mecánicas de las unidades sinterizadas se establecieron de acuerdo con la norma [21]; el ensayo universal de resistencia a la compresión se realizó en el equipo manual marca PINZUAR referencia PC 160. El ensayo de absorción se realizó siguiendo el procedimiento especificado en la norma [21].

\section{RESULTADOS Y DISCUSIÓN}

\subsection{Análisis por fluorescencia de rayos $X$}

Se observa mediante el análisis semicuantitativo por fluorescencia de rayos X (FRX) que el elemento más abundante en la muestra de arcilla es el silicio, en forma de $\mathrm{SiO}_{2}$, con un contenido es de $62,3 \%$ (ver tabla 1). Por lo que respecta al aluminio, expresado como $\mathrm{Al}_{2} \mathrm{O}_{3}$, representa el $27 \%$ de la muestra.

Tabla 1. Concentración de componentes hallados por FRX en las muestras de arcilla y cenizas volantes.

\begin{tabular}{|c|c|c|}
\hline Componente & $\begin{array}{c}\text { Muestra cenizas } \\
\text { (\%) }\end{array}$ & $\begin{array}{c}\text { Muestra } \\
\text { arcilla (\%) }\end{array}$ \\
\hline $\mathrm{Al}_{2} \mathrm{O}_{3}$ & - & 27 \\
\hline $\mathrm{SiO}_{2}$ & 88,7 & 62,3 \\
\hline $\mathrm{K}_{2} \mathrm{O}$ & 0,75 & 1,1 \\
\hline $\mathrm{CaO}$ & 1,4 & 0,65 \\
\hline $\mathrm{TiO}_{2}$ & 2,67 & 1,2 \\
\hline $\mathrm{Fe}_{2} \mathrm{O}_{3}$ & 6,48 & 7,72 \\
\hline
\end{tabular}

Fuente: elaboración propia

La relación molar de ambos óxidos $\mathrm{SiO}_{2} / \mathrm{Al}_{2} \mathrm{O}_{3}$ en la muestra es de 2:1, aproximadamente, lo que la clasifica como una arcilla montmorillonítica [22], comprobando lo reportado en la literatura en la cual este tipo de arcilla contiene de 50 a $65 \%$ de $\mathrm{SiO}_{2}$ y de 10 a $25 \%$ de $\mathrm{Al}_{2} \mathrm{O}_{3}$ [23]. Aunque el porcentaje de $\mathrm{Al}_{2} \mathrm{O}_{3}$ es superior al del rango establecido, esta arcilla no es de alta alúmina ya que no supera el 39,6\% en composición de $\mathrm{Al}_{2} \mathrm{O}_{3}$ [24-26] considerándose como una arcilla sin propiedades refractarias.

Por otra parte, la muestra de arcilla presentó óxidos colorantes como $\mathrm{TiO}_{2}$ y $\mathrm{Fe}_{2} \mathrm{O}_{3}$ representando en conjunto $8,92 \%$, lo cual indica que este tipo de arcilla no presentará 
tonalidades blancas pues ambos elementos superan el $1 \%$ en composición requerido para mantener una coloración clara después de la cocción [27]. Debido al bajo porcentaje que representan, son compuestos trazas, y según los estudios efectuados por K. Skinner et al., y F. Wahl [28-29] estos elementos pueden facilitar la aparición de algunas fases.

En la muestra también se presentó $\mathrm{CaO}$ con un $0,65 \%$; por lo general, los carbonatos que aparecen asociados a los minerales arcillosos son calcita, dolomita y magnesita esencialmente [30-31]. Dependiendo de su composición química, las temperaturas de descomposición varían, por ejemplo, la magnesita (carbonato magnésico) se descompone a $650{ }^{\circ} \mathrm{C}$, la dolomita (carbonato cálcico-magnésico) a los $850{ }^{\circ} \mathrm{C}$ y la calcita (carbonato cálcico) a $950^{\circ} \mathrm{C}$ [32].

Propiedades similares a las del calcio las imprime también el magnesio, aunque en menor grado [33] su adición puede provocar un efecto brusco; en algunas arcillas puede actuar como elemento refractario impidiendo que se alcance la temperatura de fusión y favoreciendo la estabilización de propiedades como la porosidad y la contracción, entre otras, antes de alcanzar la fusión total [34-35].

Por otra parte, en la muestra de polvos de cenizas volantes, se encontró $\mathrm{SiO}_{2}$ óxido predominante con una presencia del $88,7 \%$ (ver tabla 1 ). Los altos contenidos de cuarzo reducen la plasticidad de las masas y su contracción durante el secado; además, permiten controlar la porosidad y, a la vez, aumentar la velocidad de secado de las unidades cerámicas moldeadas [36].

Dentro de los tipos de ceniza aceptados por norma (UNE-EN 197-1:2000), la muestra se caracteriza por ser ceniza volante silícea; como se evidencia en los trabajos de M. Frías et al. [37], este tipo de ceniza posee un contenido de $\mathrm{CaO}$ reactivo menor al $10 \%$.

La ceniza de carbón debido a su composición química puede ser utilizada en la gran mayoría de pastas cerámicas, ya que posibilita la fabricación de unidades más livianas [38-39], además de una mejor aislación termo-acústica [40].

\subsection{Límites de Atterberg}

En cuanto al mínimo porcentaje de agua que se debe agregar a la mezcla para la fabricación de las unidades cerámicas macizas, se realizaron ensayos de plasticidad en la muestra de arcilla considerando que cada mineral de este suelo presenta un grado diferente según sus características cristal-químicas. De los tipos de arcillas existentes, la de mayor plasticidad es la montmorillonita mientras que la caolinita es considerada una de las menos plásticas, junto con la illita [41-44]. 
Los resultados de cada uno de los ensayos realizados a la muestra de arcilla se indican en la tabla 2 , observándose un $40,5 \%$, para el límite líquido, y para el límite plástico, un $15,45 \%$. Una vez se han determinado los límites se puede hallar el índice de plasticidad; usando la ecuación (a) este parámetro dio 15\%.

Tabla 2. Propiedades: límite líquido, límite plástico e índice de plasticidad de la muestra de arcilla.

\begin{tabular}{|l|c|}
\hline \multicolumn{1}{|c|}{ Ensayos } & (\%) \\
\hline Límite líquido (LL) & 40,5 \\
\hline Límite plástico (LP) & 15,45 \\
\hline Índice de plasticidad (IP) & 15 \\
\hline
\end{tabular}

Fuente: elaboración propia

Con el diagrama de Casagrande o carta de plasticidad se puede definir si la plasticidad en la muestra de arcilla es alta, media o baja [45]. Como se puede observar en la figura 1 se ubicó el límite líquido (LL) y el índice de plasticidad (IP). El punto en el plano queda por encima de la línea A, lo cual permite deducir que el material se caracteriza por ser una arcilla inorgánica de plasticidad media a baja (CL).

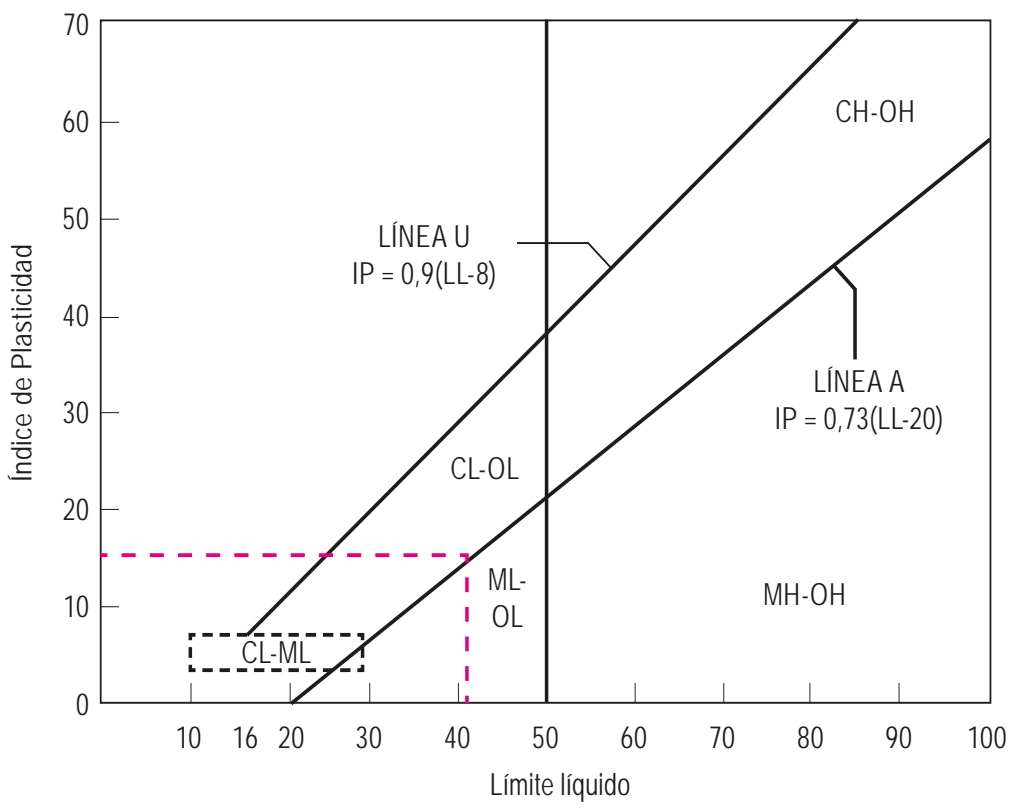

Figura 1. Carta de plasticidad para la clasificación de suelos finos.

Fuente: elaboración propia

El porcentaje de agua que se agregó a la mezcla para elaborar las piezas fue de $20 \%$ ya que con esta cantidad se presentaron condiciones de plasticidad adecuadas 
para el tipo de conformado; además, el porcentaje de agua añadido se encuentra dentro del rango entre el límite plástico y el límite líquido.

\subsection{Propiedades mecánicas}

El ensayo universal de resistencia a la compresión de las unidades elaboradas, siguió el procedimiento señalado en la norma NTC 4205. Como se puede observar en la tabla 3 , al comparar las cinco unidades a base de arcilla y cenizas volantes (de referencia) con las unidades con adición de $\mathrm{MgSO}_{4} \cdot 7 \mathrm{H}_{2} \mathrm{O}$, se puede afirmar que la presencia de un alto contenido de sales en la matriz no afecta de manera importante la resistencia a la deformación ante un esfuerzo de compresión, ya que cuatro probetas de las cinco propuestas superaron la resistencia mínima exigida por norma.

Tabla 3. Resultados ensayo universal de resistencia a la compresión de las unidades cerámicas macizas de referencia y las unidades con adición de $\mathrm{MgSO}_{4} \cdot 7 \mathrm{H}_{2} \mathrm{O}$.

\begin{tabular}{|c|c|c|c|}
\hline \multirow{2}{*}{$\begin{array}{l}\text { Resistencia unidades referencia } \\
\qquad\left(\mathrm{kg} / \mathrm{cm}^{2}\right)\end{array}$} & \multirow{2}{*}{$\begin{array}{l}\text { Resistencia unidades con } \\
\mathrm{MgSO}_{4} \cdot 7 \mathrm{H}_{2} \mathrm{O}\left(\mathrm{Kg} / \mathrm{cm}^{2}\right)\end{array}$} & \multicolumn{2}{|c|}{ Norma } \\
\hline & & Unidad & Promedio \\
\hline 170,15 & 166,34 & \multirow{5}{*}{\multicolumn{2}{|c|}{$\begin{array}{l}150 \mathrm{~kg} / \mathrm{cm}^{2} \\
200 \mathrm{~kg} / \mathrm{cm}^{2}\end{array}$}} \\
\hline 155,24 & 177,21 & & \\
\hline 164,3 & 123,7 & & \\
\hline 151,1 & 150,61 & & \\
\hline 153,47 & 151,3 & & \\
\hline
\end{tabular}

Fuente: elaboración propia

\subsection{Absorción}

Las unidades de mampostería deben cumplir con las especificaciones para la absorción máxima de agua señaladas en la tabla 4; allí se puede observar que la presencia de sales no generó ningún efecto en los porcentajes de absorción. Al determinar los promedios de absorción de las cinco probetas de referencia y las cinco con adición de $\left(\mathrm{MgSO}_{4}\right.$ • $7 \mathrm{H}_{2} \mathrm{O}$ ) se comprueba que ambos lotes de ladrillos se encuentran en norma para uso como mampostería estructural exterior o interior.

\subsection{Análisis morfológico}

Una vez se conformaron y secaron las unidades cerámicas se presentó una eflorescencia total (velo de secadero) en la superficie, como se puede observar en la figura 2. Esto ocurre cuando las manchas son generadas por la presencia de magnesio, el cual produce desprendimiento de polvos superficiales de color blanco tanto en el exterior como en el interior de los ladrillos [46]. 
Tabla 4. Resultados ensayo de absorción máxima de agua de las unidades cerámicas macizas de referencia y las unidades con adición de $\mathrm{MgSO}_{4} \cdot 7 \mathrm{H}_{2} \mathrm{O}$.

\begin{tabular}{|c|c|c|c|}
\hline $\begin{array}{c}\text { Absorción unidades referencia } \\
(\%)\end{array}$ & $\begin{array}{c}\text { Absorción unidades } \\
\text { con } \mathrm{MgSO}_{4} \cdot 7 \mathrm{H}_{2} \mathrm{O}(\%)\end{array}$ & \multicolumn{2}{|c|}{ Norma } \\
& 16,24 & Unidad & Promedio \\
\hline 14,72 & 17,15 & \multirow{2}{*}{$16 \%$} & \\
\hline 13,47 & 11,22 & & \\
\hline 14,74 & 14,54 & \\
\hline 14,68 & 15,98 & & \\
\hline 14,75 & & & \\
\hline
\end{tabular}

Fuente: elaboración propia

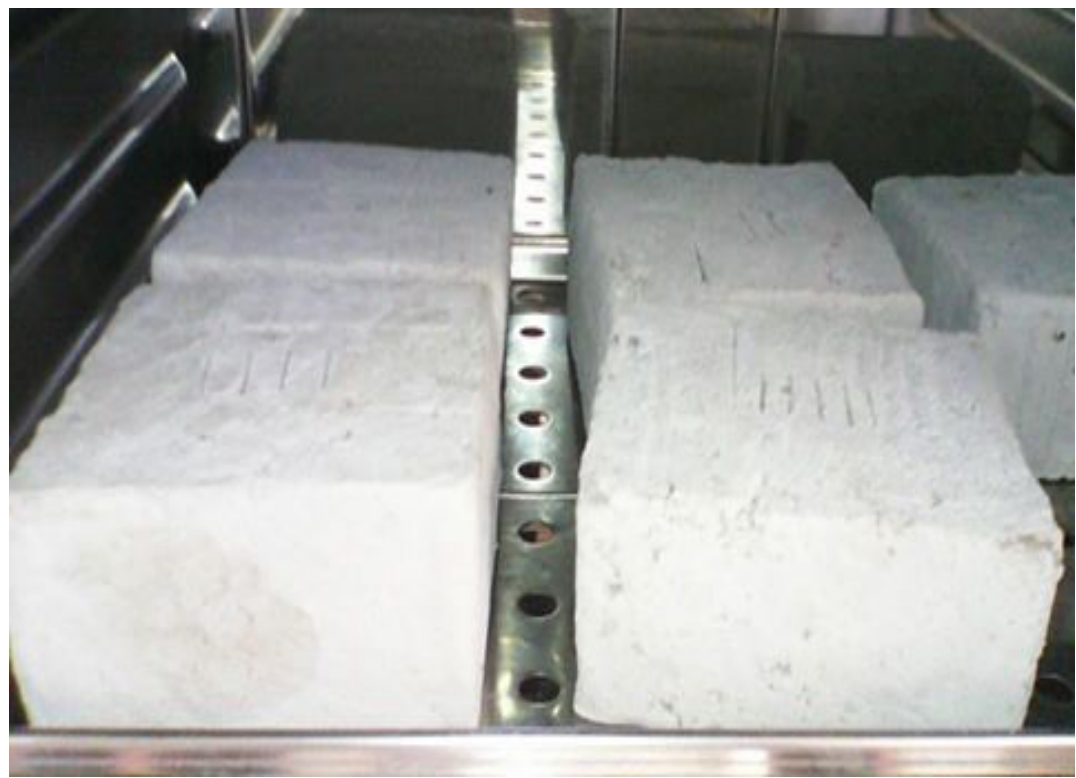

Figura 2. Unidades cerámicas macizas con eflorescencia total en la superficie (velo de secadero).

Fuente: elaboración propia

Después del proceso de sinterizado se realizó un corte transversal en una de las probetas cerámicas (ver figura 3); allí se pudo observar la capa blanca que recubría los ladrillos en una vista de perfil. En la figura, se puede ver una franja con tonalidad más clara (región A) la cual indica un alto contenido de sulfato de magnesio. Esta franja se presenta debido a que los suelos arcillosos tienen la capacidad de hidratarse tanto en la superficie como en sus espacios interlaminares y el agua que se absorbe se dispone de una manera ordenada, siendo más perfecta a medida que se acerca a la superficie [47]. 


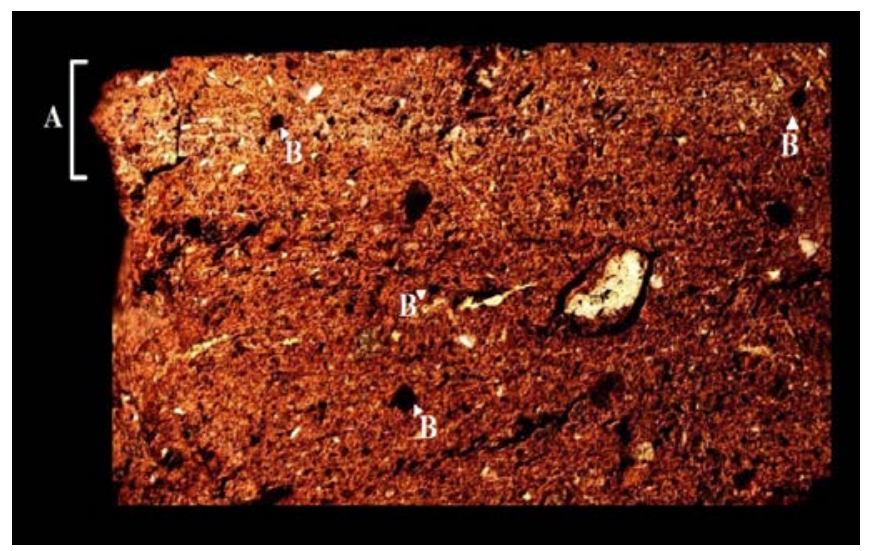

Figura 3. Corte transversal probeta cerámica sinterizada.

Fuente: elaboración propia

Debido a que el sulfato de magnesio estaba completamente disuelto en el agua, la capa indica la dirección de salida del líquido, el cual fue dejando altas concentraciones de sal en la parte donde la humedad se ubicó más ordenadamente (superficie).

La presencia de hierros y sulfuros en la mezcla generó pirita $\mathrm{FeS}_{2}$ (B) la cual también crea eflorescencias [48] y desconchados en la superficie [49].

Los polvos generados en la superficie de las unidades cerámicas macizas fueron analizados por fluorescencia de rayos X (ver figura 4). En la muestra se encontraron cuatro elementos que pueden llegar a formar sales solubles: sodio $(\mathrm{Na})$, magnesio $(\mathrm{Mg})$, potasio (K) y calcio (Ca). Por otro lado, se hallaron elementos como titanio (Ti), hierro (Fe), aluminio (Al), silicio (Si) y azufre (S).

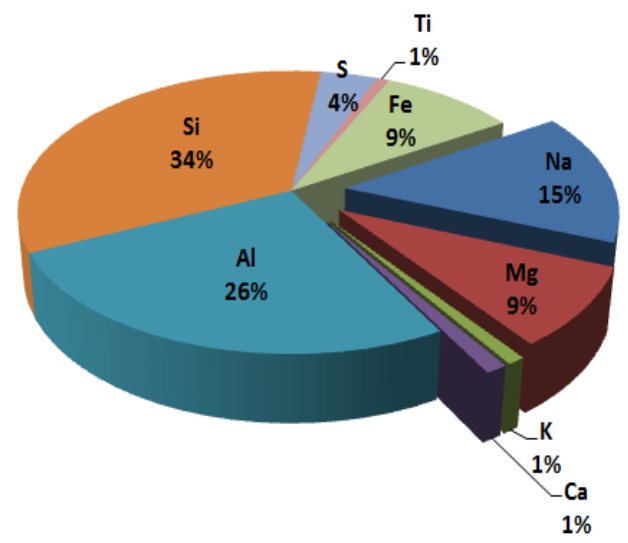

Figura 4. Concentración de componentes hallados por FRX de polvos de eflorescencia superficial en las unidades cerámicas macizas. 
En los polvos de la superficie se encontró un $15 \%$ de sodio que demuestra que los elementos que pueden llegar a formar las eflorescencias no necesariamente deben ser los mismos que se encuentran presentes en las materias primas [46]. Por otra parte, el magnesio representó el 9\% de la muestra. Este elemento en la matriz no solo fue causante de eflorescencias, sino que pudo haber facilitado la formación de fase cordierita durante el proceso de sinterización a $900{ }^{\circ} \mathrm{C}$ [50]. El calcio, el potasio y el titanio representaron $1 \%$ cada uno; azufre, silicio, hierro y aluminio se encontraron con $4 \%, 34 \%, 9 \%$ y $26 \%$, respectivamente.

Es relevante señalar la presencia en el laboratorio de un velo de horno blanco durante el proceso de cocción. Este velo es una capa de reacción originada por la interacción entre la capa salina y los componentes composicionales de las unidades cerámicas [46]. Su aparición al nivel industrial representa un gran problema debido a que puede causar eflorescencias en el lote de ladrillos. Para combatir este fenómeno se requiere la aplicación de sales que insolubilicen los sulfatos de magnesio y calcio, tales como fosfato trisódico, carbonato de sodio y carbonato de bario en proporciones de $0,25 \%, 0,25 \%$ y $0,5 \%$, respectivamente $[51,11]$.

\section{CONCLUSIONES}

El análisis por fluorescencia de rayos X (FRX) de la muestra de arcilla manifestó que el elemento más abundante es $\mathrm{SiO}_{2}$, con un contenido de $62,3 \%$, y $\mathrm{Al}_{2} \mathrm{O}_{3}$ con el $27 \%$. La relación molar de ambos óxidos es de 2:1 aproximadamente, lo que la clasifica como una arcilla montmorillonítica.

Los ensayos de plasticidad realizados reportan que para el límite líquido se obtuvo 40,5\% y para el límite plástico, un 15,45\%. El índice de plasticidad y el límite líquido caracterizan el material como una arcilla inorgánica de plasticidad media a baja (CL).

Se presentaron condiciones de plasticidad adecuadas en la mezcla para el tipo de conformado con un porcentaje de agua de $20 \%$, el cual se encuentra dentro del límite líquido y plástico del material arcilloso.

De las cinco piezas cerámicas elaboradas a base de arcilla, cenizas volantes y sulfato de magnesio, cuatro superaron la resistencia mínima a la compresión exigida por norma. Al comparar con la resistencia de las unidades de referencia, se concluye que la presencia de un alto contenido de sales no afecta de manera importante la resistencia a la deformación de las probetas ante un esfuerzo de compresión.

La absorción de agua de las unidades no se vio afectada significativamente por la adición de sulfato de magnesio, ya que al determinar los promedios de absorción de ambos lotes de ladrillos se pudo observar que cumplen con el porcentaje máximo admitido por norma, para mampostería estructural ya sea de uso interno o externo. 


\section{AGRADECIMIENTOS}

A la Dirección de Investigación de la UPTC (DIN) que mediante el proyecto Capital Semilla SGI 1997 financió la presente investigación.

Al Instituto de Recursos Minero Energéticos (Irme) por toda la asesoría y guía prestada durante el proceso de investigación.

Al Instituto para la Investigación e Innovación en Ciencia y Tecnología de Materiales (Incitema) por los servicios de laboratorios.

\section{REFERENCIAS}

[1] E. Juárez Badillo y A. Rico Rodríguez, Mecánica de suelos. México: Limusa, 1996.

[2] G. Reeves et al., "Clay materials used in construction," London: Geological Society of London, 2006.

[3] J. Martín Martín et al., Mineralogía de arcillas cerámicas. Castellón de la Plana: Universitat Jaume I, 2005.

[4] I. Bernal de Ramírez et al., "Análisis próximo de arcillas para cerámica," Revista de la Academia Colombiana de Ciencias Exactas, Físicas y Naturales, 27, pp. 569-578, 2003.

[5] J. Morales Güeto, Tecnología de los materiales cerámicos. Madrid: Díaz de Santos, 2005.

[6] D. Askeland, y G. García, Ciencia e ingeniería de los materiales. México: International Thomson Editores, 1998.

[7] M. Carretero León y M. Pozo Rodríguez, Mineralogía aplicada. Madrid: Thomson-Paraninfo, 2007.

[8] S. Crespo Escobar, Materiales de construcción para edificación y obra civil. 2010.

[9] M. Barreda et al., "Determinación de sales solubles y eflorescencias en tejas cerámicas", Boletín de la Sociedad Española de Cerámica y Vidrio, 49(3), pp.189-196, 2010.

[10] C. Grossi y R. Esbert, "Las sales solubles en el deterioro de rocas monumentales. Revisión bibliográfica”, Materiales de Construcción, 44(235), pp.15-30, 1994.

[11] J. Rincón y M. Romero, "Prevención y eliminación de eflorescencias en la restauración de ladrillos de construcción”, Materiales de Construcción, 51(261), pp. 73-78, 2001.

[12] P. López-Arce, "Daños por cristalización de sales. En: La conservación de los geomateriales utilizados en el patrimonio”, Programa Geomateriales, pp. 97-105, 2012.

[13] J. Osuna, Estudio general sobre la eflorescencia en obra. Frupesa. Madrid, 1998.

[14] S. Grimán et al., "Influencia de las variables de procesamiento tecnológico industrial en la aparición del defecto de eflorescencia en piezas de arcilla cocida”, Revista Politécnica, 36(1), 2015. 
[15] F. Lasheras Merino, "Actas del $4^{\circ}$ Congreso de Patología y Rehabilitación de Edificios. Patorreb 2012”, en $4^{\circ}$ Congreso de Patología y Rehabilitación de Edificios. Patorreb, Madrid, 2012.

[16] A. García Verduch y V. Sanz Solana, Velos, florescencias y manchas en obras de ladrillo. Castellón: Faenza Editrice Ibérica, 1999.

[17] J. González et al., "Concentración de vanadio y níquel en cenizas volantes por combustión controlada”, Interciencia, vol. 29, n. 9, pp. 504-509, 2004.

[18] I. Iglesias, A. Acosta, E. García Romero y J. Rincón, “Mejora de las propiedades cerámicas de arcillas comunes por adición de ceniza volante GICC”, en XXIV Reunión de la Sociedad Española de Arcillas, Universidad de Sevilla, 2015, pp. 31-32.

[19] Suelos. Ensayo para determinar el límite plástico e índice de plasticidad, NTC 1493, 2006.

[20] Suelos. Ensayo para determinar el límite líquido, NTC 1494, 2006.

[21] Ingeniería civil y arquitectura. Unidades de mampostería de arcilla cocida. Ladrillos y bloques cerámicos, NTC 4205, 2000.

[22] A. Alvarado, El origen de los suelos. Turrialba, Costa Rica: Catie, 1985.

[23] L. De Pablo, "Las arcillas y clasificación, identificación, usos y especificaciones industriales", Sobretiro del Boletín de la Sociedad Geológica Mexicana, 28, 1964.

[24] J. Molina, "Evaluación de mezclas de arcillas para la fabricación de ladrillos refractarios que sirvan para la reconversión tecnológica de los hornos utilizados en norte de Santander”, Revista de Investigaciones Universidad del Quindío, 1(26), 2014.

[25] J. Díaz Zamora, "Fabricación de mulita a partir de alúmina y sílice mediante molienda y variación en la composición Química”, 2015.

[26] E. Galán y P. Aparicio, "Materias primas para la industria cerámica", Seminarios de la Sociedad Española de Mineralogía, 2, pp. 31-49, 2006.

[27] A. Avgustinik, Cerámica. Barcelona: Editorial Reverté, 1983.

[28] K. Skinner et al., "Effect of TiO2, Fe2O3, and Alkali on Mineralogical and Physical Properties of Mullite-Type and Mullite-Forming A12O3-SiO2 Mixtures", Journal of the American Ceramic Society, 36(11), pp. 349-356. Thomson Editores, 1953.

[29] F. Wahl, "High-temperature phases of tree-layer clay minerals and their interactions with common ceramic materials”, Journal of the American Ceramic Society, 44, pp. 676-681, 1965.

[30] J. González et al., "La arcilla como material cerámico. Características y comportamiento", Cuadernos de Prehistoria y Arqueología de la Universidad de Granada, 8, pp. 479-490, 1983.

[31] D. Betancourt et al., "Influencia de la adición de carbonato de calcio en la eficiencia energética de la producción de ladrillos de cerámica roja”, Revista Ingeniería de Construcción, 22(3), pp. 187-196, 2007. 
[32] I. Kostov, Mineralogy. Edinburgh: Oliver \& Boyd, 1968.

[33] J. Everhart, "Use of auxiliary fluxes to improve structural clay bodies", Journal of the American Ceramic Society, 36, pp. 268-271, 1965.

[34] N. Hipedinger et al., "Comportamiento termomecánico de hormigones refractarios de liga fosfato. Influencia de los áridos en las propiedades", Actas Jornadas SAM-Conamet, pp. 977-984, 2001.

[35] J. Lasquibar y C. Ribera, "Refractarios de magnesia-carbono para cucharas", Boletín de la Sociedad Española de Cerámica y Vidrio, 28(5), pp. 385-393, 1989.

[36] R. Hevia, "Materias primas: Importancia de su conocimiento para la formulación cerámica", Cerámica y Cristal, 145, pp. 48-52, 2012.

[37] M. Frías et al., "Novedades en el reciclado de materiales en el sector de la construcción: adiciones puzolánicas”, Instituto Eduardo Torroja (CSIC), 2008.

[38] R. Loaiza y S. Steward, "Incidencia de la arcilla expandida térmicamente en los procesos de corrosión del acero de refuerzo del concreto liviano estructural”, Universidad Nacional de Colombia, 2014.

[39] M. Fratelli, Suelos, fundaciones y muros. Caracas, Venezuela: M. G. Fratelli, 1993.

[40] A. Puy Santín, "Influencia de la temperatura en el límite líquido para suelos con diferentes índices de plasticidad", M.Sc. Tesis, Universidad Politécnica de Cataluña, Barcelona, CAT, España, 2005.

[41] T. García, J. Bastida, J. De La Torre, V. Steve, T. Lores And J. Serrano, "Plasticidad y características composicionales de arcillas industriales de la zona minera de Teruel (España)", Boletín de la Sociedad Española de Mineralogía, vol. 19, n. 2 2, pp. 101-117, 1996.

[42] L. Valdez Guzmán y G. Suarez Alcivar, "Hormigones livianos”, B.S. Tesis, Escuela Superior Politécnica del Litoral, Guayaquil, GYE, Ecuador, 2010.

[43] R. Hevia, Materias primas no convencionales en cerámica. Bogotá: Fundación Emprenin, 2006.

[44] W. Cole and E. R. Segnit, "High temperature phases developed in some kaolinite-mica-quartz clays", Transactions \& Journal of the British Ceramic Society, 62, pp. 375-395, 1963.

[45] B. M. Das, Principios de ingeniería de cimentaciones. 4ta edición. México: International, 2001.

[46] J. M. Rincón and M. Romero, "Basis and classification of efflorescences in construction bricks”, Materiales de Construcción, 50(260), pp. 63-69, 2000.

[47] R. T. Martin, “Absorbed water on clays: A review”, Clays Clay Miner, 9, pp. 28-71, 1962.

[48] E. Amrein, "La lucha contra las eflorescencias", Materiales de Construcción, 11(104), 1961. 
[49] R. B. Montoro, "Terms used in the Pathology and Restoration of Brick Masonry", Revista Electrónica, 4, pp. 47-50, 2014.

[50] J. B. C. Airan, "Petrografía y Mineralogía de arcillas en relación con procesos de cocción rápida", Boletín de la Sociedad Española de Cerámica y Vidrio, 21, pp. 15-22, 1982.

[51] A. G. Verduch, "El empleo del cuarzo en las composiciones de cerámica blanca", Boletín de la Sociedad Española de Cerámica y Vidrio, 13(5), pp. 409, 1974. 